\title{
Approach to tourism support by aerial video using CG
}

\author{
Kenji Sakoma, Noritake Seto, Kodai Miyamoto, Taketo Kamasaka, Makoto Sakamoto*, Amane Takei \\ Faculty of Engineering, University of Miyazaki, \\ Miyazaki-City, Miyazaki, Japan \\ Tsutomu Ito \\ National Institute of Technology, Ube College \\ Ube-City, Yamaguchi, Japan \\ Takao Ito \\ Graduate School of Engineering, Hiroshima University \\ Higashi-Hiroshima, Hiroshima, Japan
}

E-mail:hm16011@student.miyazaki-u.ac.jp,hm15037@student.miyazaki-u.ac.jp,hm16043@student.miyazaki-u.ac.jp,
fruits2000jp@yahoo.co.jp
*Corresponding Author

\begin{abstract}
Recently, as a first step in the tourism business, we will consider whether it is possible to increase the number of tourists by incorporating IT technologies such as CG in Introductory video. In this paper, we created a CG image of a hawk flying in the sky combined with a real Takachiho video taken with a drone. As a result, various problems have been identified, and I hope to improve them in future research to produce even better ones.
\end{abstract}

Keywords: EEG, BMI, FFT, SVM, VR

\section{Introduction}

Sightseeing is for people to refresh themselves and for their hobbies, so it heals the mind in a different environment than usual. However, from the perspective of the local people, it is a big commerce that can be expected to revitalize the area and increase the number of residents.

In recent years, rural areas have been declining due to urban concentration of work and declining birthrate and aging population. Meanwhile, the number of local governments that are trying to regain the vitality of the past by focusing on the tourism industry is increasing year by year. As a first step in the tourism industry, consider whether it is possible to increase the number of tourists by incorporating IT technologies such as CG into (a) The 2021 International Conference on Artificial Life and Robotics (ICAROB2021), January 21 to 24, 2021 introductory videos such as PV and commercials.

This research is part of a joint development with Takachiho Town, and the beautiful nature of Takachiho is created by making the images taken by the drone look like the mountain hawk-eagle inhabiting Takachiho is swirling or plunging in the sky. We conducted basic research to create a promotional video to introduce people in the area.

\section{Benefits of tourism ${ }^{4}$}

When many tourists come to visit due to the development of the tourism industry, economic activities in various fields such as accommodation, transportation, eating and drinking, and travel industry become active, and the economic ripple effect is high. For example, by 
utilizing the nature and historic sites that originally existed in the area, even a small scale can be established as an industry. Therefore, at least some funds can be developed. Also, if you can attract tourists from abroad, you can earn foreign currency. In particular, in Takachiho Town, the mythical nature is the centerpiece of tourism, so even a small amount of assets can demand high productivity.

Initiatives of Takachiho Town ${ }^{5}$.

- A tour of mythical nature and shrines (see Fig. 2).

- PR of traditional Takachiho Kagura based on myth (see Fig. 1).

- Sale of gourmet foods and souvenirs that make use of the rich land of Takachiho Town.

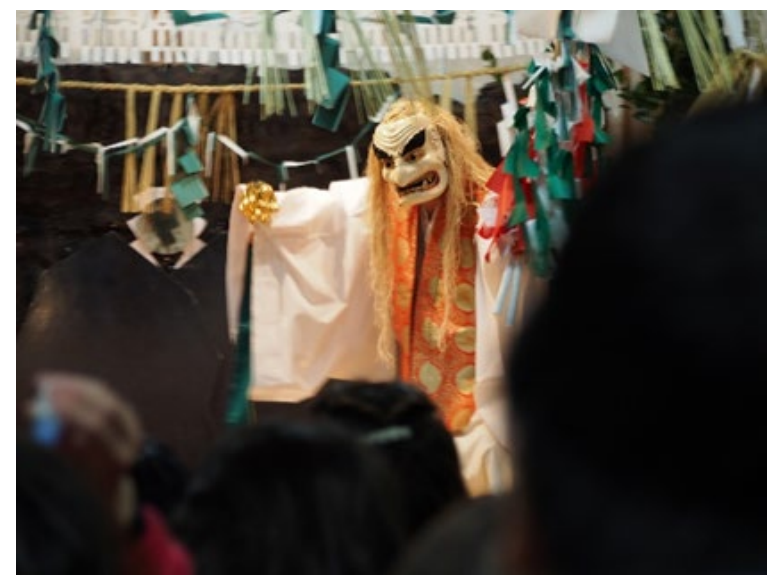

Fig. 1. Actual Kagura.

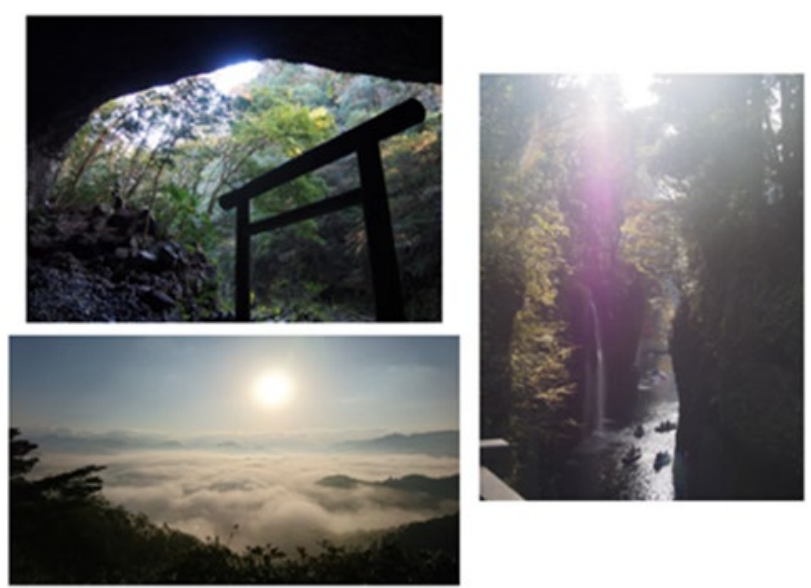

Fig. 2. Amanoyasukawara, Kunimigaoka, Takachiho Gorge.

\section{3. $\mathbf{C G}$}

In a narrow sense, it refers to images and videos generated from scratch through drawing, editing, and processing on a computer, and in a broader sense, it is a composite of images created by a computer with the original photographs, drawings, and videos. Including those processed and processed in a manner significantly different from the original $\left.{ }^{6}\right)$

In recent years, AR (Augmented Reality) and VR (Virtual Reality) have made remarkable progress. However, CG is playing an active role not only in entertainment, but also in invisible parts such as model production of architectural design and simulation in the event of a disaster.

\section{CG Tool}

In this research, Blender is used for CG creation, and Unity is used for CG operation control.

\subsection{Blender ${ }^{8}$}

Objects are created from a single cube using the extrusion and split functions (see Fig. 3). In order to smoothly reproduce the flapping of the wings, which is the greatest feature of birds, a total of 12 bones, 6 on each side from the base to the tip of the wings, are added to play the role of joints. Bones are normal ones and Bbones that are easier to express curves.

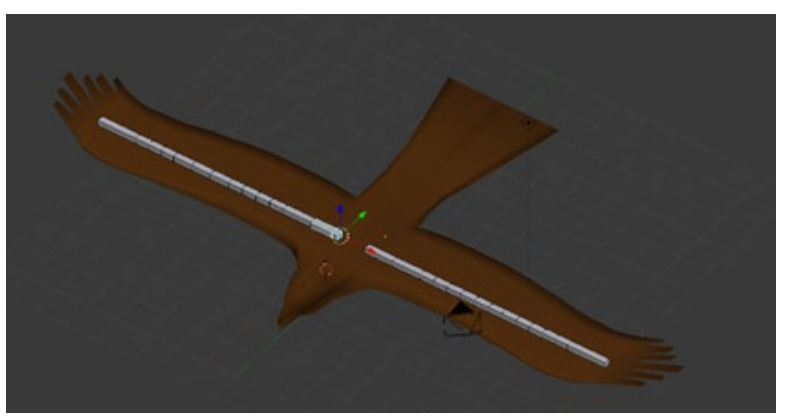

Fig. 3. The created hawk.

\subsection{Unity ${ }^{1-3)}$}

animation

In order to express the flapping and dive of the wings, the animation function is used to assign numerical values to the main body of the hawk-eagle and the bones attached to the wings. The animation function applies keyframe animation, and by changing the interval of each numerical value, smooth movement is reproduced.

- Turning

The movement of the object turning is processed only by the script without using the animation function. In this research, since the object is moved according to

(C) The 2021 International Conference on Artificial Life and Robotics (ICAROB2021), January 21 to 24, 2021 
the video actually shot, the rotation is only rotated around the z-axis.

The turning is limited to 30 degrees on each side, and it is set so that it does not turn even if the key input is continued beyond that. However, as an important process here, the turning angle is also different between the value on the console and the value to be acquired, so conditions are set so that the value is the same.

- Image composition

An ICO ball is used to make the image change when the hawk turns.

This is achieved by installing a hawk object and a main camera inside the ICO sphere (see Fig. 4) so that the sphere rotates when the arrow keys are input.

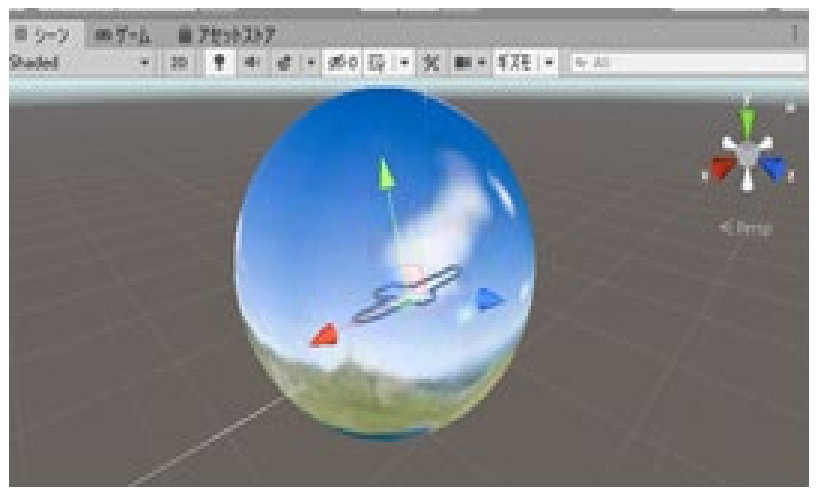

Fig. 4. Composition of 360-degree image on a sphere - ICO sphere

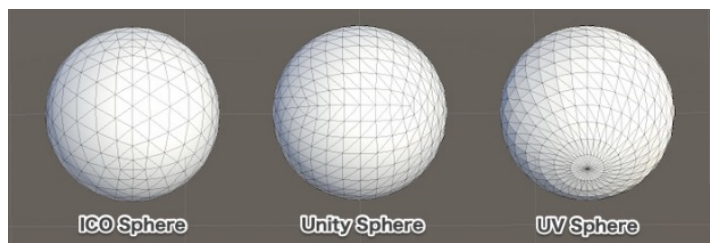

Fig. 5. 3D sphere model.

In this research, since it is necessary to paste the texture without distortion as much as possible, ICO spheres are used in the 3D sphere model (see Fig. 5), and ICO spheres are treated as distributed ${ }^{9)}$.

- glTF
Blender can export in glTF format, which allows it to be imported into Unity with almost the same shading as Blender (see Fig. 6).

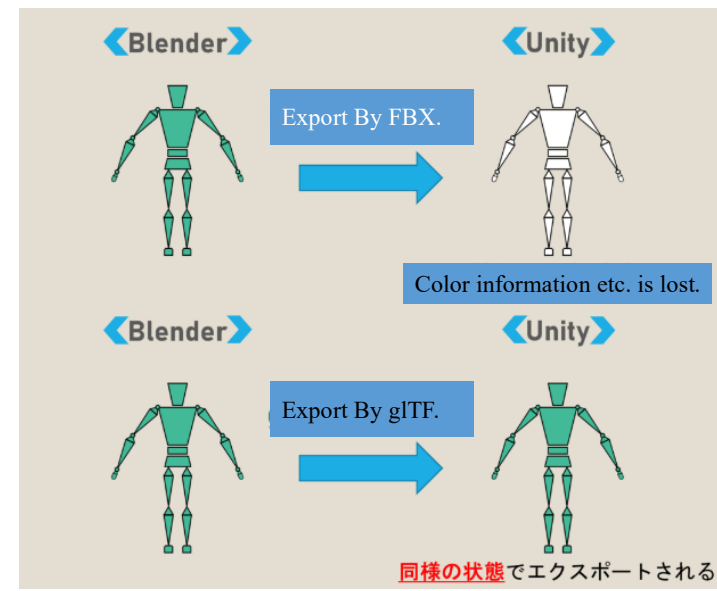

Fig. 6. glTF

Since it is necessary to paste the image on the inner surface of the sphere, download and use the UniGLTF plug-in ${ }^{10)}$.

- Screen transition due to a sudden descent of a hawk

The screen transition is processed by the script, and the screen transition is performed when the space key is input.

In this state, the screen transition will be performed before the animation of the sudden descent of the mountain hawk eagle is performed, so when the space key is input, the elapsed time will be observed and the video of the tourist introduction will be played when the elapsed time reaches the end of the animation. Transition to the scene.

- Sound / Video

Free materials are used for sounds, hawk-eagle calls, aerial images, and sightseeing introduction videos 7).

\section{Consideration / Future issues}

In this research, we used Blender and Unity to create a CG animation that promotes Takachiho by synthesizing the CG of a mountain hawk eagle flying in the sky with the actual image of Takachiho taken aerial with a drone. This time, we succeeded in pasting the image on the sphere, but there is a problem that the resolution of the free and usable image is too low and it becomes too rough at the time of compositing. 
In addition, although the specification was originally to combine images, the moving image file must be in the Equirectangular format in order to combine images from a 360-degree viewpoint, so in this research, images are combined.

Since it is possible to transition to only one scene in the screen transition, I think that it will be possible to transition to multiple scenes by acquiring the rotation angle of the sphere and assigning it to the scene corresponding to a specific angle.

\section{References}

1. Mikito Yoshito, "Unity5 3D/2D Introduction to game development practice", Soshimu, 2015.

2. "Ministry of Internal Affairs and Communications Information and Communications White Paper Internet penetration (2015 version)",

[Online].

http://www.soumu.go.jp/johotsusintokei/whitepaper/ja/h27 $/ \mathrm{html} / \mathrm{nc} 372110 . \mathrm{html}$.

3. "Unity DOCMENT/Unity Manual", [Online]. https://docs.unity3d.com/ja/current/Manual/index.html.

4. "Tourism Wikipedia", [Online]. https://ja.wikipedia.org/wiki/\%E8\%A6\%B3\%E5\%85\%89 $\% \mathrm{E} 6 \% \mathrm{~A} 5 \% \mathrm{AD}$.

5. "Takachiho Kankokyokai Official site", [Online]. http://takachiho-kanko.info/.

6. "IT Yogo jiten e-Words CG", [Online]. http://e-words.jp/w/CG.html.

7. "On-Jin $\sim$ Onjin ", [Online].http://onjin.com/sound/kan.php?kate=\%E9\%A2\%A8.

8. "Blender.jp", [Online]. https://blender.jp/.

9. "360-dgree eizo no gosei",

[Online]. https://shinrinmusic.com/unity-360-image/.

10. "gITF",

[Online]. https://xr-hub.com/archives/20285. 